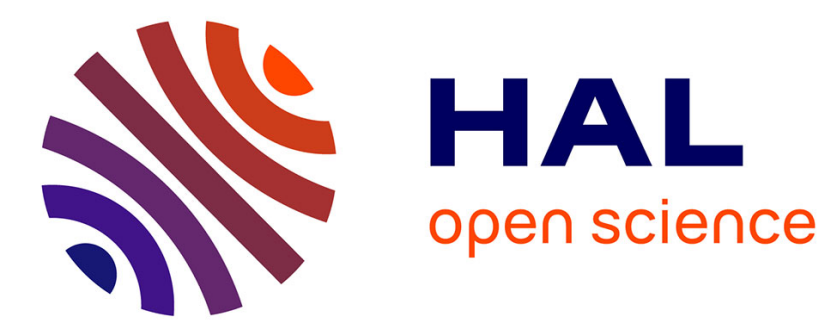

\title{
On droplets size distribution in a pulsed column. Part I: In-situ measurements and corresponding CFD-PBE simulations
}

\author{
A. Amokrane, S. Maass, F. Lamadie, François Puel, S. Charton
}

\section{To cite this version:}

A. Amokrane, S. Maass, F. Lamadie, François Puel, S. Charton. On droplets size distribution in a pulsed column. Part I: In-situ measurements and corresponding CFD-PBE simulations. Chemical Engineering Journal, 2016, 296, pp.366-376. 10.1016/j.cej.2016.03.089 hal-01301432

\section{HAL Id: hal-01301432}

https://hal-centralesupelec.archives-ouvertes.fr/hal-01301432

Submitted on 14 Jun 2018

HAL is a multi-disciplinary open access archive for the deposit and dissemination of scientific research documents, whether they are published or not. The documents may come from teaching and research institutions in France or abroad, or from public or private research centers.
L'archive ouverte pluridisciplinaire HAL, est destinée au dépôt et à la diffusion de documents scientifiques de niveau recherche, publiés ou non, émanant des établissements d'enseignement et de recherche français ou étrangers, des laboratoires publics ou privés. 
This Accepted author version posted online: 14 June 2018. Published online: 26 March 2016

To cite this article: A. Amokrane, S. Maab, F. Lamadie, F. Puel, S. Charton (2016) On Droplets Size Distribution in a pulsed column: Part I : In-situ measurements and corresponding CFD-PBE simulation, Chemical Engineering Journal 296, 366-376 , DOI: /10.1016/i.cej.2016.03.089

\title{
On Droplets Size Distribution in a pulsed column. Part I: In-situ measurements and corresponding CFD-PBE simulations.
}

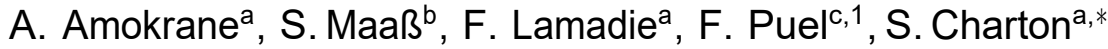 \\ ${ }^{a}$ CEA, DEN, DTEC, SGCS, F-30207, Bagnols-sur-Cèze, France \\ ${ }^{b}$ SOPAT GmbH, D-10587, Berlin, Germany \\ 'Université de Lyon, Université Lyon 1, CNRS, CPE Lyon, UMR 5007, Laboratoire \\ d'Automatique et de Génie des Procédés (LAGEP), 43 Bd du 11 Novembre 1918, \\ F-69622 Villeurbanne, France
}

\begin{abstract}
The pulsed column is one of the most used contactors in solvent extraction processes, especially in the nuclear fuel treatment industry and for hydrometallurgical applications. The optimization of solvent extraction operations needs a thorough understanding of the diphasic flows properties inside the apparatus, especially that of the dispersed phase. For the first time, in-situ measurements of the drop size distribution (DSD) in a small diameter pulsed column (lab scale) were achieved by using the SOPAT-VF light reflectance video probe on two water in oil (W/O) systems exhibiting low and high viscosities of the oil phase. Separate effects of the pulse amplitude and frequency on the DSD and on the phase hold-up were evidenced for both $\mathrm{O} / \mathrm{W}$ system, despite their different viscosity levels. This technique allowed very precise measurements of droplets diameter, over three orders
\end{abstract}

\footnotetext{
*Corresponding author: sophie.charton@cea.fr

${ }^{1}$ Current affiliation: Laboratoire de Génie des Procédés et Matériaux, CentraleSupélec, Université Paris Saclay, Grande Voie des Vignes, 92295 Châtenay-Malabry, France
} 
of magnitude, in a quite confined space, which is remarkable. Experimental findings allowed the validation of a computational fluid dynamics (CFD)population balance equation (PBE) coupled model and the improvement of breakage and coalescence kernel parameters. Rupture and coalescence mechanisms were correctly captured. The dependence of emulsion's properties on the column hydrodynamics could then be illustrated over one period of the pulsed flow. Incidence of higher levels of pulsation intensity, hardly achievable experimentally, and separate investigation of the effects of viscosity and surface tension are presented.

Keywords: Pulsed Column, Liquid-liquid system, DSD measurement, In-situ probe, CFD-PBE Modelling

\section{Introduction}

Predicting solvent extraction efficiency in a given apparatus requires a thorough understanding of the liquid-liquid flow in the contactor and its coupling with the chemical processes. Hence, axial mixing, dispersed phase hold-up and slip velocity (e.g. residence time ditribution) and of course interfacial area (e.g. droplets size distribution), are the main parameters of most of the models dedicated to the simulation of liquid-liquid extraction columns performances $[1,2,3,4,5]$. Moreover, coupled chemistry-transport numerical simulation became a mandatory tool for scale-up and optimization purposes, complementary and reducing the need for pilot-scale measurements, providing that the emulsification process, e.g. the tendency of the droplets to break and to coalesce, is correctly described.

However, while the disc and doughnut pulsed column is one of the most 
used industrial contactors, and is the reference one for nuclear applications, barely no data is available regarding emulsion properties in such device. Indeed, since the prior attempt of Kumar and Harland $[6,7]$ to correlate the dispersed phase properties relevant for solvent extraction in various apparatus, few studies focused on pulsed column $[2,8]$. Yet, none of them rely on sufficient or adequate test data to allow extrapolation to other column geometries or operating conditions. For other type of extractors conversly, comprehensive studies of the emulsification process are arising, that open the way to progressively more phenomelogical models (see e.g [9] for RDC columns, $[10,11]$ for centrifugal contactors, etc.).

The development of such models preferably requires on-line characterization of the dispersed phase properties. The latter are usually measured on samples taken either at the outlet or along the apparatus, generally at pilote-scale $[12,13]$. Some authors tried to measure the DSD by the outside of transparent-wall contactors using video systems and image-processing [2]. However, external monitoring is limited to low dispersed phase concentration, and non-negligible optical distorsions is usually induced by the cylindrical wall [8]. At last, in-situ measurements, using either light transmission [14], reflectance [15] or even ultrasonic [16], are more frequently dedicated to the DSD in agitated vessels. Reliability of DSD measurements is crucial for model's derivation, and many papers are dedicated to the comparison of measurement techniques $[17,18]$.

Althought large diameter pulsed columns are operated in the nuclear fuel treatment industry, size-reduction is a major issue in the scope of R\&D studies. In this aim very small diameter prototypes (ranging from 15 to $25 \mathrm{~mm}$, 
where RCD or agitated pilots are genrally $50 \mathrm{~mm}$ in diameter) are used. Because of their small hold-up, droplets characterization from sampling would be disruptive and external or in-situ techniques must be preferred. In this paper, in-situ measurements of the drop size distribution (DSD) are achieved by using a two-dimensional reflectance technique. It is the first time that such in-situ DSD data are reported for a pulsed column. Thanks to these original experimental data, the parameters of breakage and coalescence kernels relevant for typical $\mathrm{W} / \mathrm{O}$ emulsions encountered in pulsed column have been improved. The previously developped coupled fluid dynamics and population balance equations (CFD-PBE) model [19], whose main lines are briefly recalled, has been improved accordingly. The parametric studies enabled by this model provide a better understanding of the emulsification process in in the contactor, that will facilitate the transposition of the main features observed at the lab-scale to the industrial one.

\section{Material and methods}

\subsection{Setup description}

The pulsed column consists of a $25 \mathrm{~mm}$ internal diameter cylinder equipped with discs and doughnuts internals (Fig.1). The disc diameter is $21.22 \mathrm{~mm}$ and the doughnut internal diameter $12.25 \mathrm{~mm}$. The distance between a disc and a doughnut is $24 \mathrm{~mm}$. Holes are drilled on the the column wall with a spacing of $220 \mathrm{~mm}$. These holes have been used to introduce the probe in the column and to measure the hold-up by collecting small amounts of emulsion, once the steady state has been reached.

The experiments were performed using two water in oil (W/O) systems. 


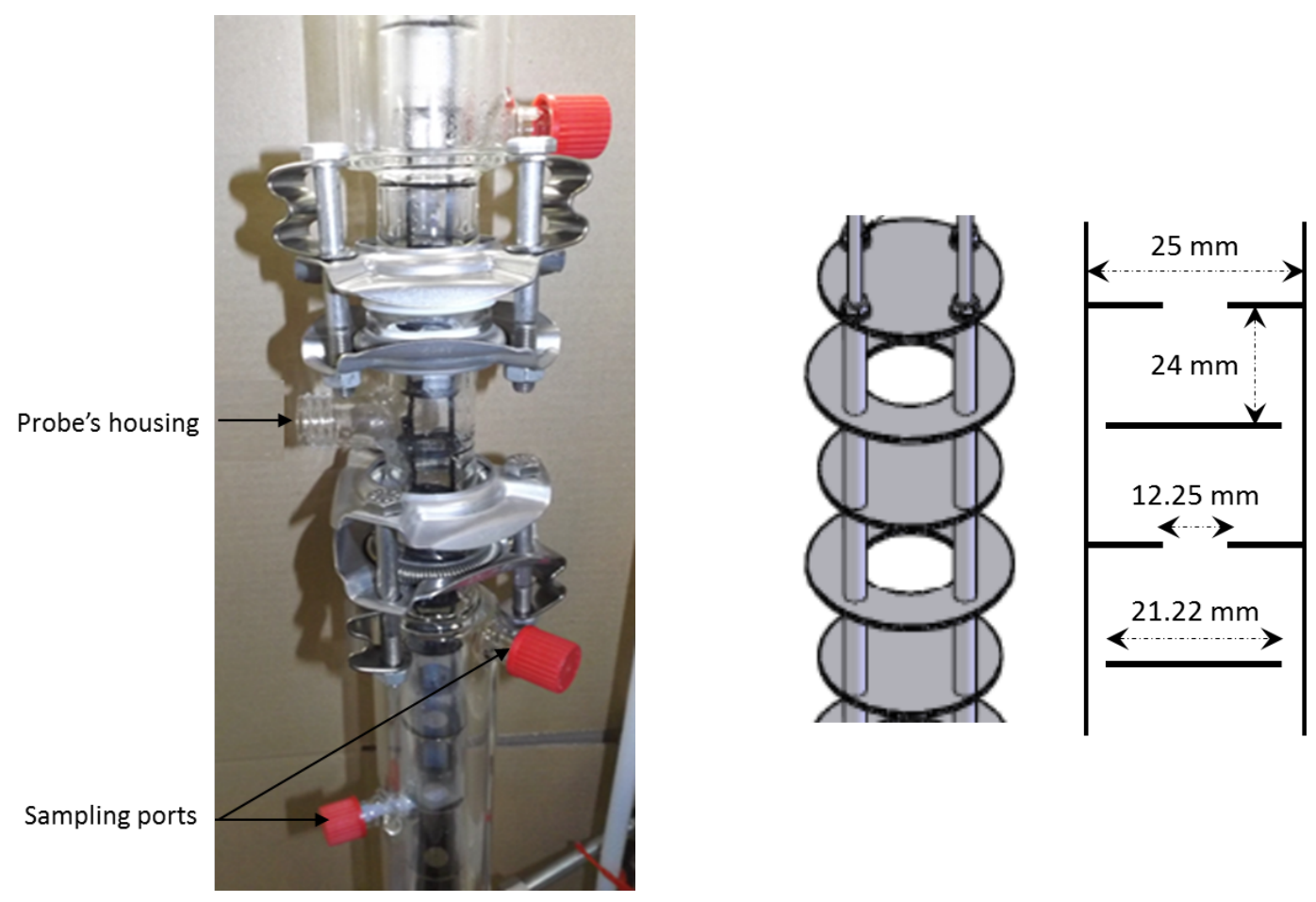

Figure 1: Left: Detail view of the pulsed column showing the housing for the video probe used for DSD measurement (diameter $16.5 \mathrm{~mm}$ ) and some of the sampling-ports used for $\phi$ measurements diameter $8.5 \mathrm{~mm}$ - Right: Sketch and dimensions of the disk and doughnut packing. 


\begin{tabular}{|c|c|c|c|c|}
\hline System & $\mathrm{O} / \mathrm{W}$ phases & $\begin{array}{c}\text { Density } \\
\left(\mathrm{kg} / \mathrm{m}^{3}\right)\end{array}$ & $\begin{array}{c}\text { Viscosity } \\
(\mathrm{Pa} . \mathrm{s})\end{array}$ & $\begin{array}{c}\text { Surface tension } \\
(\mathrm{mN} / \mathrm{m})\end{array}$ \\
\hline \multirow{2}{*}{ I } & TPH & 760 & $1.23 \times 10^{-3}$ & \multirow{2}{*}{43} \\
\cline { 2 - 4 } & Water & 998.2 & $1.10^{-3}$ & \\
\hline \multirow{2}{*}{ II } & Oil mixture & 833 & $1.38 \times 10^{-2}$ & \multirow{2}{*}{29} \\
\cline { 2 - 4 } & Nitric acid + RBS & 1050.5 & $1.0410^{-3}$ & \multirow{2}{*}{} \\
\hline
\end{tabular}

Table 1: Physical properties of the studied phase systems at $T=20^{\circ} \mathrm{C}$.

The first one (I) consists in droplets of water dispersed in Hydrogenated Tetra Propylene (TPH), a low viscosity C12 branched chain hydrocarbon. The organic phase of the second system (II) is a $(85 \%$ - 15\%) mixture of TPH and Marcol, a higher viscosity oil, while the aqueous phase is nitric acid $(1.5 \mathrm{~N})$. A water soluble surfactant, RBS, has been used to adjust the surface tension. This second liquid-liquid system allowed us to study the influence of viscosity and interfacial tension on the behaviour of the emulsion in the pulsed column. The physical properties of the two $\mathrm{W} / \mathrm{O}$ emulsion systems investigated here are gathered in Tab. 1.

The operating conditions considered in the experimental study are gathered in Tab. 2. Run 1 to 11 are referring to phase system (I), and the remaining to phase system (II) . They were selected in order to investigate the effect of the pulsation frequency and amplitude, the dispersed phase holdup,$\phi$, and of course the physical properties of the fluids, on the behaviour of the emulsion. The throughput flow-rates can be adjusted in order to vary the hold-up independently of the pulsation properties. For a given oil-inwater system, the runs were performed successively by setting the operating parameters ( $A, f$ and / or the flow-rate) to the desired value. A sufficiently long delay was systematically observed in order to ensure the stabilization of 
the emulsion. Once the DSD and hold-up were established, series of images were recorded using the probe and a small amount of emulsion was collected in order to estimate hold-up. At least 1000 droplets were detected for each measurement, except for run 9 for which only 200 droplets were detected.

\subsection{Droplets detection}

In-situ droplets monitoring has been achieved using a photo-optical device. Indeed, it is well known that externally mounted photo-optical techniques are subjected to astigmatism when applied to cynlindrical columns [20]. Additional advantage of photo-optical techniques is that backlighting is not required, as for shadowgraph based probes (e.g. as in [19]), which facilitates the detection of small droplets as well as large ones.

Photo-optical systems have been proven to be reliable by many authors in the literature (see e.g. $[21,22,23]$ ). Moreover, in oil-water systems, where droplets usually remain spherical, recent studies (e.g. in toluene-water system [18], or dark crude oils [23]) have emphasized the advantages of photooptical devices as opposed to laser-based instruments.

The SOPAT-VF measurement system used in this study is depicted in Fig 2. The probe is introduced in the pulsed column via a specific housing (see Fig.1). It is mounted in such a way that its tip is just flush with the column wall, thus minimizing the flow perturbations. However, for such an intrusive method, the possible modifications of the dispersed liquid particles flow should be kept in mind when qualitatively interpreting the measured values.

Although the optical field was reduced (by the packing elements, etc.) nice quality raw images are recorded, allowing the derivation of the DSD and 


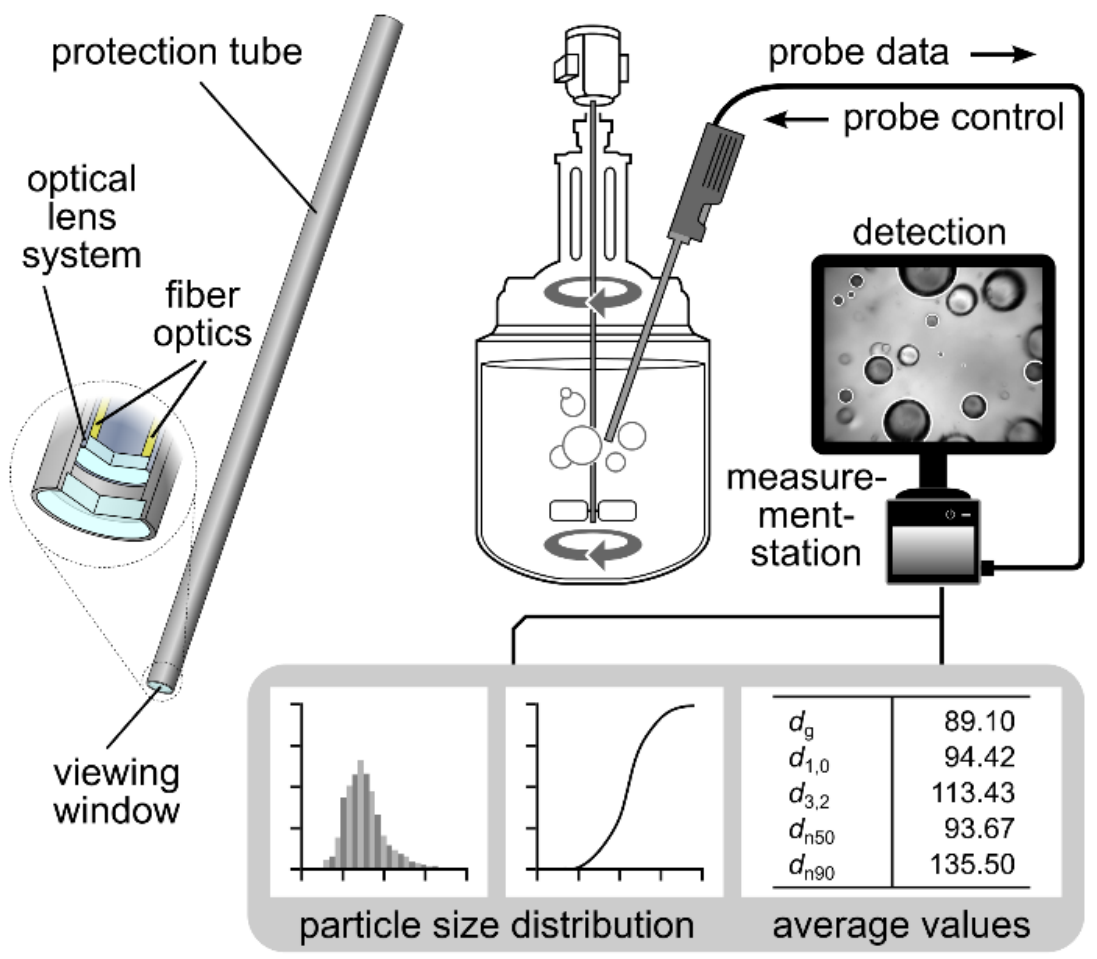

Figure 2: Sketch of the SOPAT-VF probe and principle of the measurements 

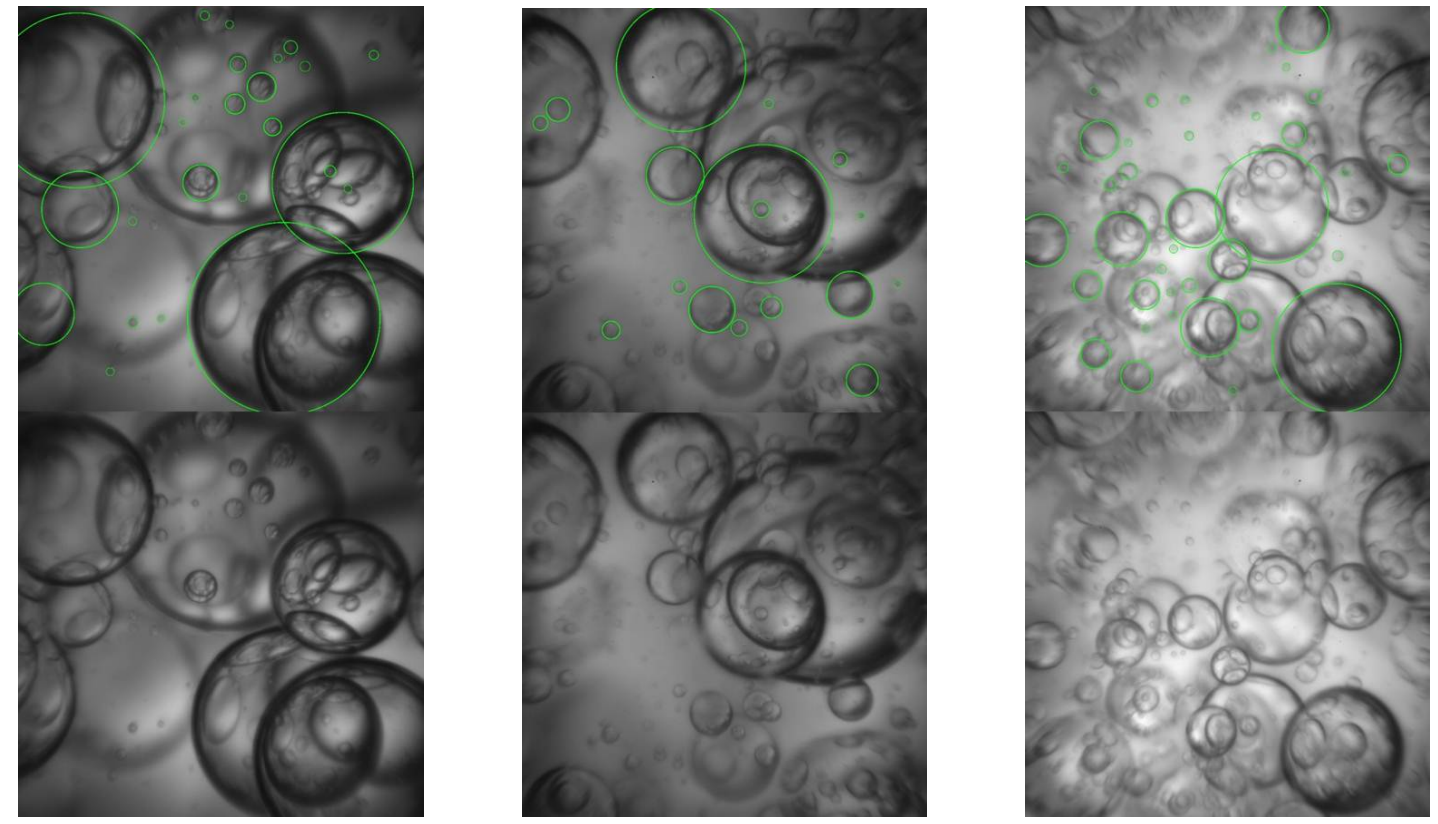

Figure 3: Example of images recorded by the video probe in the pulsed column, at increasing pulse intensity from left to right $\left(A \times f=20,40\right.$ and $\left.60 \mathrm{~mm} \cdot \mathrm{s}^{-1}\right)$. The top and bottom parts of each image are identical, except that in the top part the drops detected by the software, and that are accounted for in the experimental DSD, are circled green.

therefrom the adjustment of the breakage and coalescence kernels for the system under study. Very high quality images were also recorded in a 15-mm diameter column provided with an alternated truncated disk packing [24]. However, due to the 3D-geometry of the column, the results exploitation for modelling purpose is less convenient, and only the data measured on the 25mm columns will be considered here. An example of the images obtained is shown Fig. 3.

The principle of the droplets detection is the following. The endoscopic device projects the photographed objects onto a 6 megapixel CCD highspeed camera that can record up to 20 frames per second at full resolution. Illumination is provided by a flash (energy $2.5 \mathrm{~J}$ ) with an exposure time of 
ca. $6.5 \mu \mathrm{s}$. The measure is based on the reflection of incident light: the light provided by the optical fiber is redirected by the interfaces between the drops and the continuous phase, thanks to the difference in refraction index between the two fluids. Data acquisition, storage and post-processing is performed by a computer.

The capability of the photo-based endoscope technique was previously demonstrated by means of a sensitivity analysis of the changes in Sauter mean diameter $\left(d_{32}\right)$ in times in a stirred batch reactor, for various specific power input (see Maaß et al. [18]).

Many researches are currently focused on the development of automated drop detection and analysis from digitized images. Image processing is generally based on Hough algorithm [25] or morphology operations [26]. However, most of these studies deals with dispersed phase fraction significantly lower than $5 \%$, where only little overlapping of the drops occurs [14, 21]. Recently Mickler et al. [27] were able to increase the dispersed phase concentration up to $15 \%$, by using distance transformation and watershed segmentation algorithms.

The SOPATR software for image recognition is based on the works of Alban et al. [28] and Brás et al. [29] who considered up to $40 \%$ concentrated dispersions in stirred vessels. Robust and accurate drop detection is ensured by first pre-filtering the serie of images to remove irrelevant and misleading information. This is achieved by subtraction of an integrated sequence. The noise in the pictures is then reduced by the self quotient image method [30]. This operation norms the intensity of every local pixel based on the local environment. It is carried out by division of the processed image by a 
smoothed version of itself. Then the drop recognition is achieved. It consists of three steps: i) pattern recognition by correlation of pre-filtered gradients with search samples, ii) pre-selection of plausible circle coordinates and iii) classification of each of those circles by an exact edge examination. The software employs a normalized cross correlation procedure [31] to evaluate possible drop matches. This fast and efficient automated image analysis algorithm avoids the usual limitations of image-processing (circular shape, no overlapping, etc.). See Maaß et al. [15] for more technical details and results.

\subsection{Emulsion's properties modelling: breakup and coalescence kernels}

The breakup and coalescence kernels proposed by Coulaloglou and Tavlarides [32] were used to account for the modification undergone by the dispersed phase due to breakup and coalescence phenomena. These models are well known and widely used in the literature.

The breakup kernel, Eq. (1), is a phenomenological one. It states that the energy supplied by the turbulence eddy needs to overcome the surface energy of the droplet for the collision to be efficient.

$$
b\left(d_{i}\right)=C_{1} d_{i}^{-2 / 3} \frac{\varepsilon^{1 / 3}}{1+\phi} \exp \left[-\frac{C_{2} \sigma(1+\phi)^{2}}{\rho_{d} \varepsilon^{2 / 3} d_{i}^{5 / 3}}\right]
$$

Although the model accounts for the influence of the fluids and the flow properties on the breakup frequency $\left(\rho_{d}\right.$ and $\phi$ stand respectively for the dispersed phase density and concentration, $\sigma$ is the interfacial tension, and $\epsilon$ the mean turbulent dissipation), the constants $C_{1}$ and $C_{2}$ are not universal ones and numerical values need to be assigned to fit either the phase system 
or/and the hydrodynamic conditions (see e.g. [33, 34, 35]).

Together with the breakup model described below, and based on the first observations of the DSD in the pulsed column, the Valentas model, Eq. (2), has been selected for the daughter drops distribution [36].

$$
\beta\left(d_{1}^{3} \mid d_{0}^{3}\right)=\frac{2.4}{d_{0}^{3}} \exp \left(-\frac{4.5\left(2 d_{1}^{3}-d_{0}^{3}\right)^{2}}{d_{0}^{6}}\right)
$$

where $d_{0}$ and $d_{1}$ stand for the mother and the daughter droplet diameter respectively. This model assumes binary breakup.

The coalescence model describes the coalescence frequency as the product of a collision frequency, $h\left(d_{i}, d_{j}\right)$, between two drops of diameter $d_{i}$ and $d_{j}$, and the efficiency of this collision, $\lambda\left(d_{i}, d_{j}\right)$. The collision frequency is due to the random turbulent fluctuations, resembling to the random walk of gas molecules in the kinetic theory of gases. The collision frequency is hence expressed as:

$$
h\left(d_{i}, d_{j}\right)=C_{3}\left(d_{i}+d_{j}\right)^{2}\left(d_{i}^{2 / 3}+d_{j}^{2 / 3}\right)^{1 / 2} \epsilon^{1 / 3}
$$

The collision efficiency relies on the film drainage theory, that compares the contact time of the two droplets to the one required, under the lubrification theory, for the thin film drainage:

$$
\lambda\left(d_{i}, d_{j}\right)=\exp \left(-\frac{t_{\text {drainage }}}{t_{\text {contact }}}\right)=\exp \left[-C_{4} \frac{\mu_{c} \rho_{c} \epsilon}{\sigma^{2}}\left(\frac{d_{i} d_{j}}{d_{i}+d_{j}}\right)^{4}\right]
$$

As for breakage, the two constants $C_{3}$ and $C_{4}$ of the coalescence model are assigned experimental values. 


\subsection{Coupled CFD-PBE model}

The numerical model, developed under ANSYS-FLUENTR, was introduced in a previous paper [19].

The computational domain consists of 6 compartments, e.g. 6 successive disc-doughnut-disc patterns, in a 2D axis-symmetric framework. The mesh, refined near the walls and internals, counts 36,600 cells (see Appendix 1).

The fluid flow description is based on the $k-\epsilon$ turbulence model, coupled with a First Order Upwind scheme, that appeared to predict correctly the turbulent dissipation $\epsilon$ under pulsed flow conditions [37]. A periodic velocity profile of amplitude $A$ and frequency $f$, is imposed as the boundary condition for the continuous phase in one side of the computational domain, while a fixed pressure outlet condition is used on the opposite side.

Due to the low concentration of droplets considered here, the conclusions of the one-phase flow studies can be transposed to the two-phase case. Besides, the drag coefficient is modeled by the Schiller and Naumann correlation.

The dispersed phase evolution is described by a population balance equation $(\mathrm{PBE})$ in a Euler-Euler frame. The PBE is solved by the QMOM method $[38,39]$ considering the six first moments $m_{i}$ of the DSD. Knowledge of the initial droplet volume fraction $\phi$ and of the diameter of the water primary droplets is sufficient for determining the initial value of the first moment $m_{0}$. The following five initial moments $m_{1}, . ., m_{5}$ are deduced by recursion, as described in [39]. As the simulation was performed, the mean Sauter diameter $d_{3,2}$ was calculated considering the $m_{2}$ and $m_{3}$ moments.

The convergence study based on the dispersed phase properties, indicated 
that the appropriate time-step $d t$ for the coupled CFD-PBE simulations is ten times smaller that the value appropriate for the one-phase simulations, resulting from the Courant criteria $(d t=1 \mathrm{~ms})$. Hence, all simulations were performed considering $d t=0.1 \mathrm{~ms}$. All simulations were run in parallel on 4 cores, and convergence was obtained in approximatly 20 hours depending on the considered operating conditions.

\section{Results and discussion}

The results of the experimental study are first described, and the emulsion sensitivity to the column operating conditions is discussed. Then experimental and numerical results are compared for relevant experiments for validation purpose. In the last part, some complementary simulations are discussed in order to further investigate the droplets behaviour in the pulsed column.

\subsection{Experimental results}

The mean diameter $\left(d_{32}\right)$ and dispersed phase fraction $(\phi)$ that were measured at equilibrium for each investigated operating conditions are indicated in Tab. 2.

As expected, we can observe from Fig. 4 that increasing the pulsation intensity results in a decrease of the Sauter diameter $d_{32}$, and consequently an increase of the interfacial area. This is for instance evidenced by the transition between successive runs 1 and 2, or successive runs 3 and 4, for which the $A \times f$ was increased from 40 to $60 \mathrm{~mm} \cdot \mathrm{s}^{-1}$ in phase system (I). This effect is observed regardless of the liquids properties, as evidenced by the transition between runs 12 and 15 relative to phase system (II), where $A \times f$ is

increased from 15 to $37 \mathrm{~mm} . \mathrm{s}^{-1}$, all other parameters being unchanged. This 


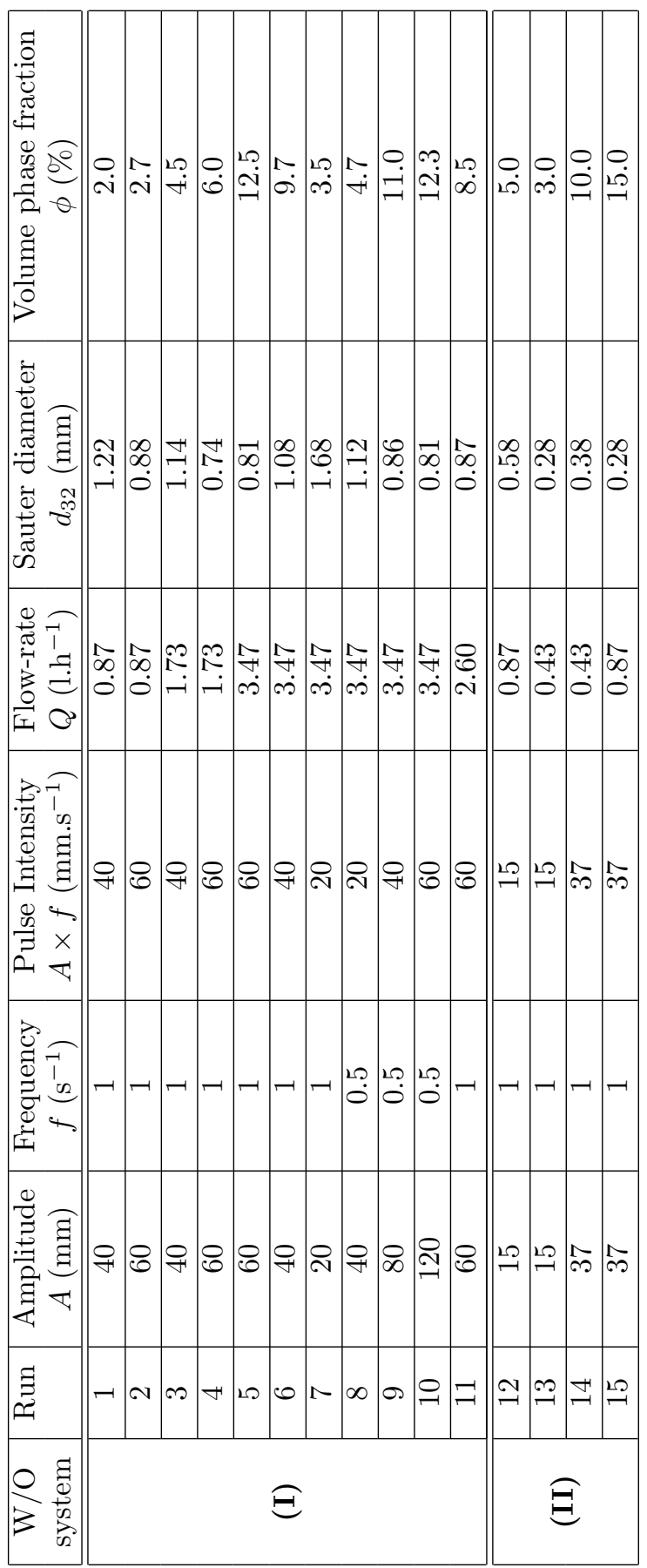

Table 2: Operating conditions for the experimental study and corresponding emulsion properties. The flow-rate is relative to the dispersed (aqueous) phase, which was varied to fix the phase ratio. Experiments were generally run without axial flow of the continous phase. For phase system (II) however, tiny 1 droplets were formed and a small throughput flow-rate was imposed $\left(2.7\right.$ l.h $\left.{ }^{-1}\right)$ to avoid flooding. 


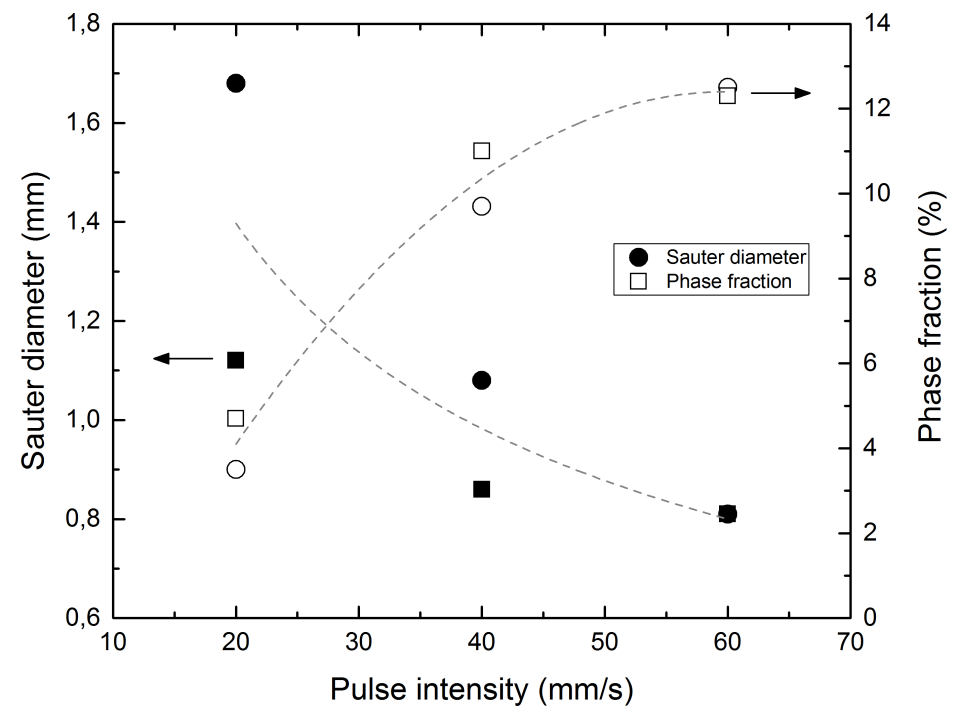

Figure 4: Measured evolution of $d_{32}$ (black symbols) and $\phi$ (white symbols) with increasing pulse intensity, at $f=1$ (circles) and 0.5 (squares) $\mathrm{s}^{-1}$. The lines show overall trends $\left(Q=3,471 . \mathrm{s}^{-1}\right.$ for all experiments).

evolution of $d_{32}$ reflects a higher comminution of the emulsion, as evidenced by the translation of the whole DSD to the left, illustrated by Fig. 5. The reverse is of course verified too. Hence, as evidenced from the results of the transition $(5 \mapsto 6)$, decreasing the pulse intensity induces a growth of the drops, due to breakage dampening.

The experimental results also highlighted an effect of the pulsation intensity on the concentration of droplets, $\phi$ (e.g. the hold-up). Hence, keeping the flow-rate, $Q$, constant, a higher $A \times f$ results in a higher $\phi$, presumably due to more fragmentation and appearance of small droplets with greater residence time in the compartment.

On the other hand, little information can be deduced regarding the in- 


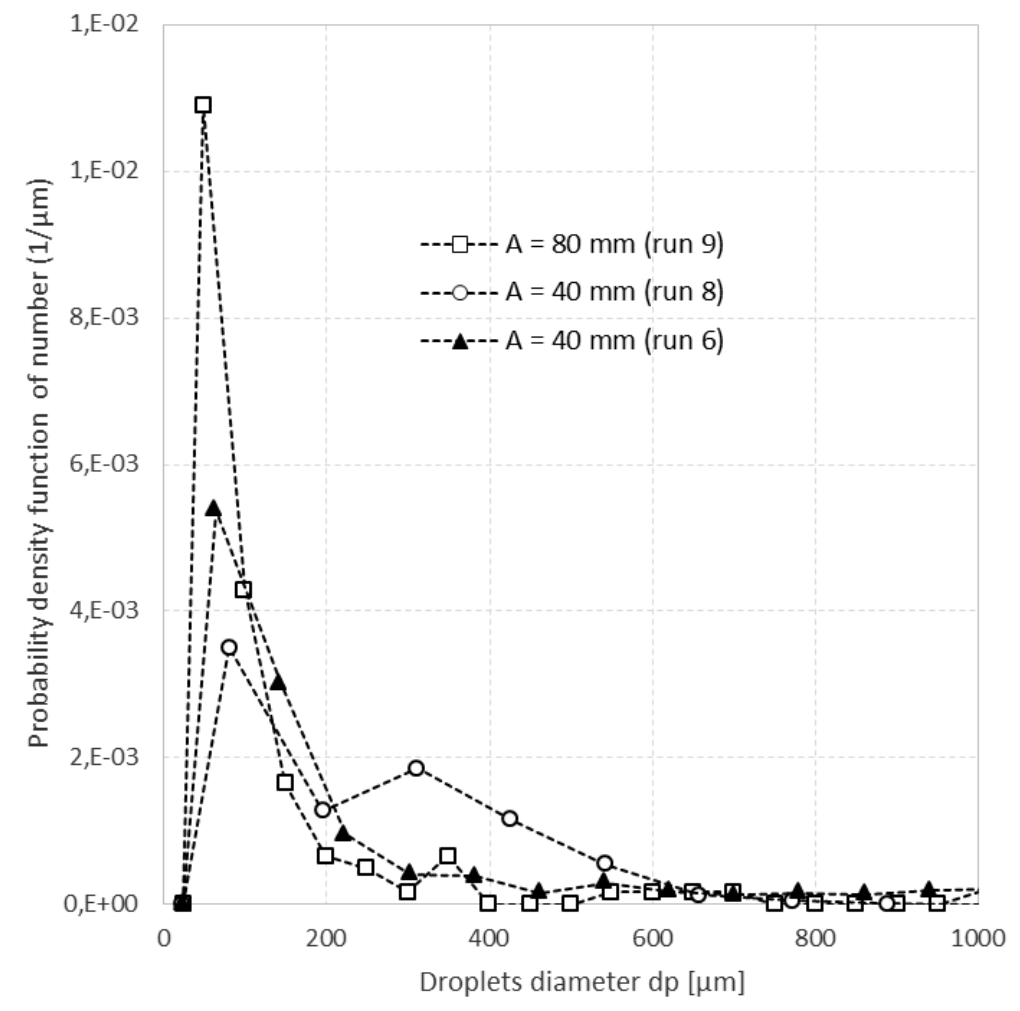

Figure 5: DSD comparison at different operating conditions for phase system $\mathbf{I}(Q=3.47$ $1 . h^{-1}$ in all cases). The increased effect of fragmentation at higher intensity induces a more efficient comminution of the droplets (runs 8 and 9 at the same $f=0.5 \mathrm{~s}^{-1}$ ). Comparing runs 6 and 9 highligths the separate effect of $A$ and $f$ (same intensity $40 \mathrm{~mm} . \mathrm{s}^{-1}$ ). 
fluence of the concentration of droplets on the interfacial area, since $\phi$ was generally fixed by the feed flow-rate $Q$. However, a slight decrease of the mean drop diameter is observed at higher rentention, although this effect is not as pronounced as the pulsation intensity one, and the explaination less obvious. It has been observed too that the higher the pulse intensity the lower the effect, that is nearly negligible at $60 \mathrm{~mm} \cdot \mathrm{s}^{-1}$.

The pulse amplitude and frequency are observed to have a separate effect on the emulsion properties. Indeed, if we compare experiments 7 and 8 , or 6 and 9 (see also Fig. 5) that were carried out at the same intensity $(A \times f=20$ and $40 \mathrm{~mm} \cdot \mathrm{s}^{-1}$ respectively) and same feed flow-rate, a smaller $d_{32}$ is achieved for the higher amplitude case. Again, this tendency appears to be damped by the pulse intensity, and reveals negligible at $60 \mathrm{~mm} \cdot \mathrm{s}^{-1}$ (runs 5 and 10 respectively).

At last, the effect of the fluid properties can be studied by comparing the behaviour observed with the two $\mathrm{W} / \mathrm{O}$ dispersions, that differ by the viscosity of the continuous phase and the surface tension (see Tab. 1).

Because of their different properties, it was not possible to operate the column under the exact same conditions for phase system (II) than for phase system (I). Although comparable pulsation intensitie were considered, high pulsation amplitude $(A<40 \mathrm{~mm})$ couldn't be explored with phase system (II). Indeed, as reported in Tab. 2, a thinner emulsion was systematicaly achieved, and due to a sharp increase of the phase fraction, a net throughput flow of the continous phase had to be established to address flooding issues. Typical DSD achieved for phase systems (I) and (II) are compared on Fig 6.

Studies dedicated to the influence of the viscosity of the continuous phase 

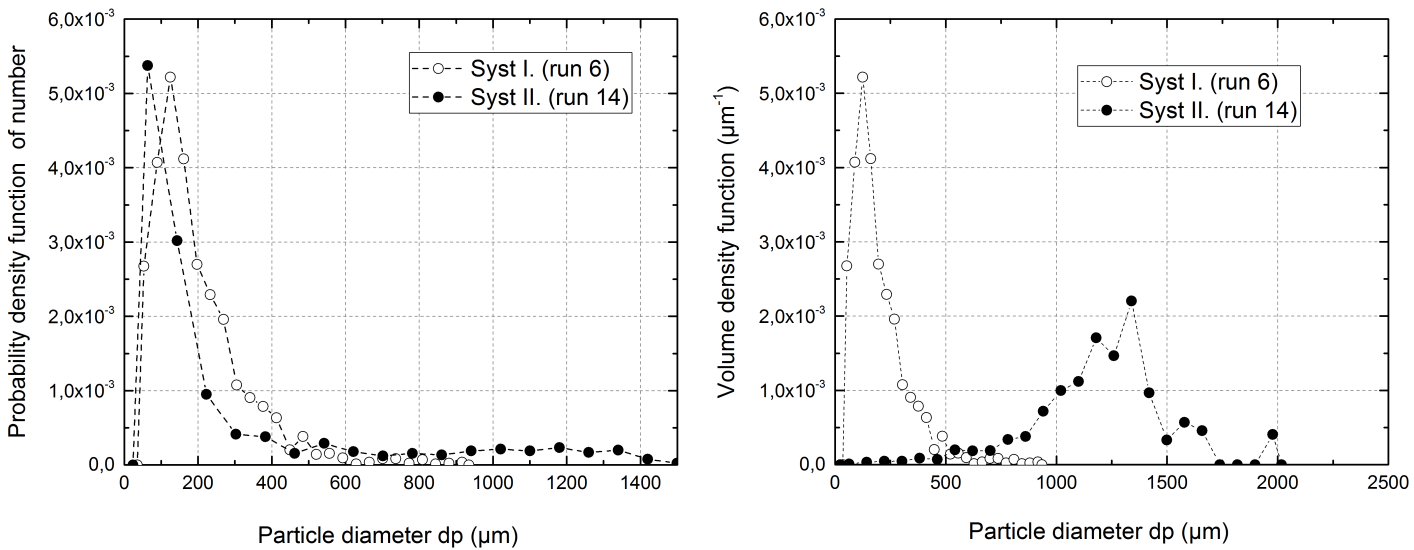

Figure 6: Comparison of the DSD measured for phase system I (Run 6) and phase system II (Run 14). Left: Number density function - Right: Volume (i.e. mass) density function. Both experiments were carried under similar operating conditions $\left(A \times f \approx 40 \mathrm{~mm} \cdot \mathrm{s}^{-1}\right.$ and $\phi \approx 10 \%$ ).

are rather uncommon. Indeed, most studies focus on oil-in-water emulsions $[35,40]$. They reveal that increasing the viscosity of the dispersed phase results in the appearance of more and more big droplets, and a subsequent change in the shape of the DSD. The latter indeed becomes larger and a distinct secondary peak appears in the small droplets region, leading to a bimodal DSD.

Although such a DSD enlargement was not clearly evidenced by our measurements, it is worth mentioning that $i)$ very large droplets $(d>2 \mathrm{~mm})$ were visually observed while considering the high viscosity continous phase, although the latter were not detected by the probe (thus explaining the DSD truncation on Fig. 6 left), and ii) the proportion of tiny droplets is bigger (as evidenced on Fig. 6 right). The latter are indeed responsible for the flooding problems encountered with phase system (II), and which led us to 
superimpose an axial flow of the continuous phase to advected the fines upward. This result is consistent with the phenomena depicted in [41], where breakup is assumed to increase with the viscosity of the continuous phase. However, one should keep in mind that, regardless of the net axial flow, the flow regimes are different for both liquid-liquid systems. In fact, the pulsation Reynolds number, $R e=(2 A f D) / \nu,{ }^{2}$ is ranging from 45 to 112 for the viscous fluid (system (II)), while it is between 100 and 1800 for first system. Hence, it is likely that the breakup mechanisms differ for the two systems, thus preventing us from further comparison.

\subsection{Kernels parameters identification}

In a previous paper [19], we presented an identification procedure for the determination of breakup and coalescence kernels constants, based on the dynamic evolution of the DSD in a stirred-tank reactor. The experiments were performed in a range of rotating rate achieving mean turbulent dissipation $\epsilon$ in the vessel similar to the one prevailing in the pulsed column. However, by using the thus-fitted coefficients in the PBE-CFD simulations, a systematic discrepancy was observed between the predicted and measured values of $d_{32}$ and $\phi$, likely due to differences in the flow anisotropy. An adjustment of the model parameters was therefore required. The latter was not based on the dynamic evolution of the DSD, as we did before, since it would have required the application of an inverse method on the CFD-PBE coupled model. The following simplified procedure was implemented instead (see Appendic 2):

\footnotetext{
${ }^{2} R e$ is typically defined considering $2 A f$, the mean periodic velocity of the oscillating flow, and $D$ the column diameter as the characteristic dimension.
} 


\begin{tabular}{|c|c|c|c|}
\hline$C_{1}$ & $C_{2}$ & $C_{3}$ & $C_{4}$ \\
\hline \hline 0.0803 & 0.0635 & $4.5 \times 10^{-2}$ & $1.89 \times 10^{11}$ \\
\hline
\end{tabular}

Table 3: Adjusted paramaters of the Coulaloglou and Tavalrides model [32], based on W/0 phase system I, and relevant for the pulsed column.

1. the initial state is set from the equilibrium achieved fo given operating conditions ;

2. the simulation of the next experimental conditions is performed;

3. the constants related to the coalescence / breakage probability, $C_{1}$ and $C_{3}$, are adjusted through a series of iterative simulations until the best agreement between the simulated and the measured $d_{32}$ is achieved ;

4. the other model parameters, $C_{2}$ and $C_{4}$, related to the efficiency of these mechanisms, are then adjusted ;

5. iterations are made until the best fitting was reached.

A series of successive experiments performed with the $\mathrm{W} / \mathrm{O}$ phase system (I) was considered in this purpose: i) successive runs $1 \mapsto 2$ and $i i$ ) successive runs $3 \mapsto 4 \mapsto 5 \mapsto 6 \mapsto 7 \mapsto 8$. The other experiments were not considered as they either were not consecutive, thus not knowing their initial state, or regarding the viscous phase system (II), because we selected turbulent kernels that are not supposed to be relevant at low Re, as previously discussed.

The numerical values identified for $C_{1}, C_{2}, C_{3}$ and $C_{4}$ are reported in Tab. 3.

In order to assess the model ability to handle both breakage and coalescence dominated cases, numerical and experimental results are compared in Table 4 for operating conditions corresponding to either an increase or a decrease of the pulsation intensity. 


\begin{tabular}{|c|c|c|c|c|}
\hline \multirow{2}{*}{ Sequence } & \multicolumn{2}{|c|}{$d_{32}(\mathrm{~mm})$} & \multicolumn{2}{c|}{$\phi(\%)$} \\
\cline { 2 - 5 } & Mesurement & Simulation & Measurement & Simulation \\
\hline $40 \mapsto 60 \mathrm{~mm} . \mathrm{s}-1$ & 0.74 & 0.84 & 6.0 & 5.6 \\
\hline $60 \mapsto 40 \mathrm{~mm} . \mathrm{s}-1$ & 1.08 & 0.89 & 9.7 & 9.5 \\
\hline
\end{tabular}

Table 4: Comparison between experimental and numerical results for a breakage dominate system (run $3 \mapsto$ run 4 ) and a coalescence dominated one (run $5 \mapsto \operatorname{run} 6$ ).

\subsection{Parametric sensitivity, simulation results}

All the simulations have been performed using the adjusted parameters. While the DSD derived from the in-situ observations of the droplets are indicative of the population in the compartment on average, simulation results give insights on the local distribution and therefrom about how the column internal geometry influences the emulsification process. An example of coupled CFD-PBE simulation result is given Fig. 7, where the mean diameter, $d_{32}$ of the emulsion is plot at different instants of the pulse period. Fig. 8 gives additionnal illustration of the coupling between the column hydrodynamics and the emulsion's properties.

\section{Influence of the pulsation intensity}

The model ability to predict the effect of the pulsation intensity on the emulsion's properties is highlighted by Fig. 9. In accordance with the experimental findings, the higher the pulse intensity the lower the mean diameter, and the higher the dispersed phase hold-up.

\section{Individual effect of amplitude and frequency}

The pulsation intensity, $A \times f$, is usually considered as the parameter of relevance to characterize the hydrodynamics of the pulsed column in the 

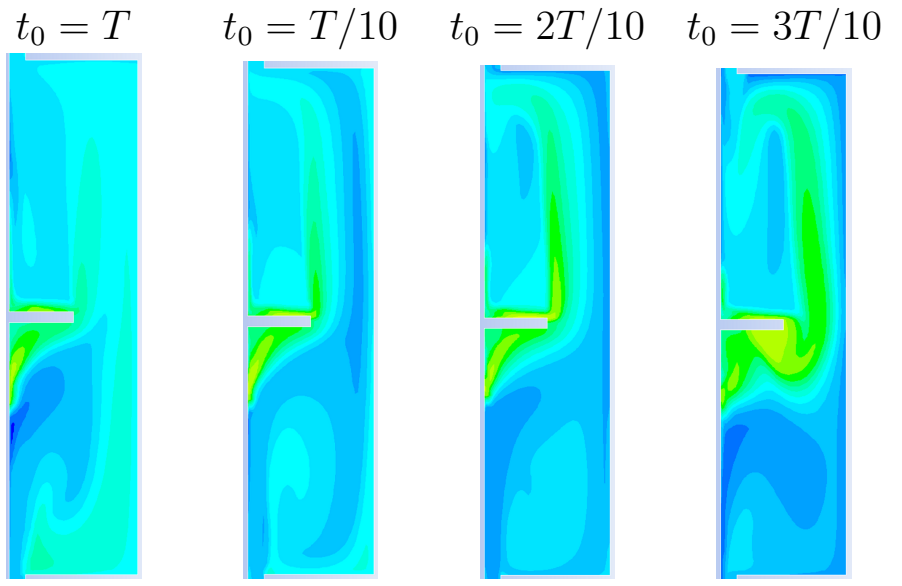

$t_{0}=4 T / 10$
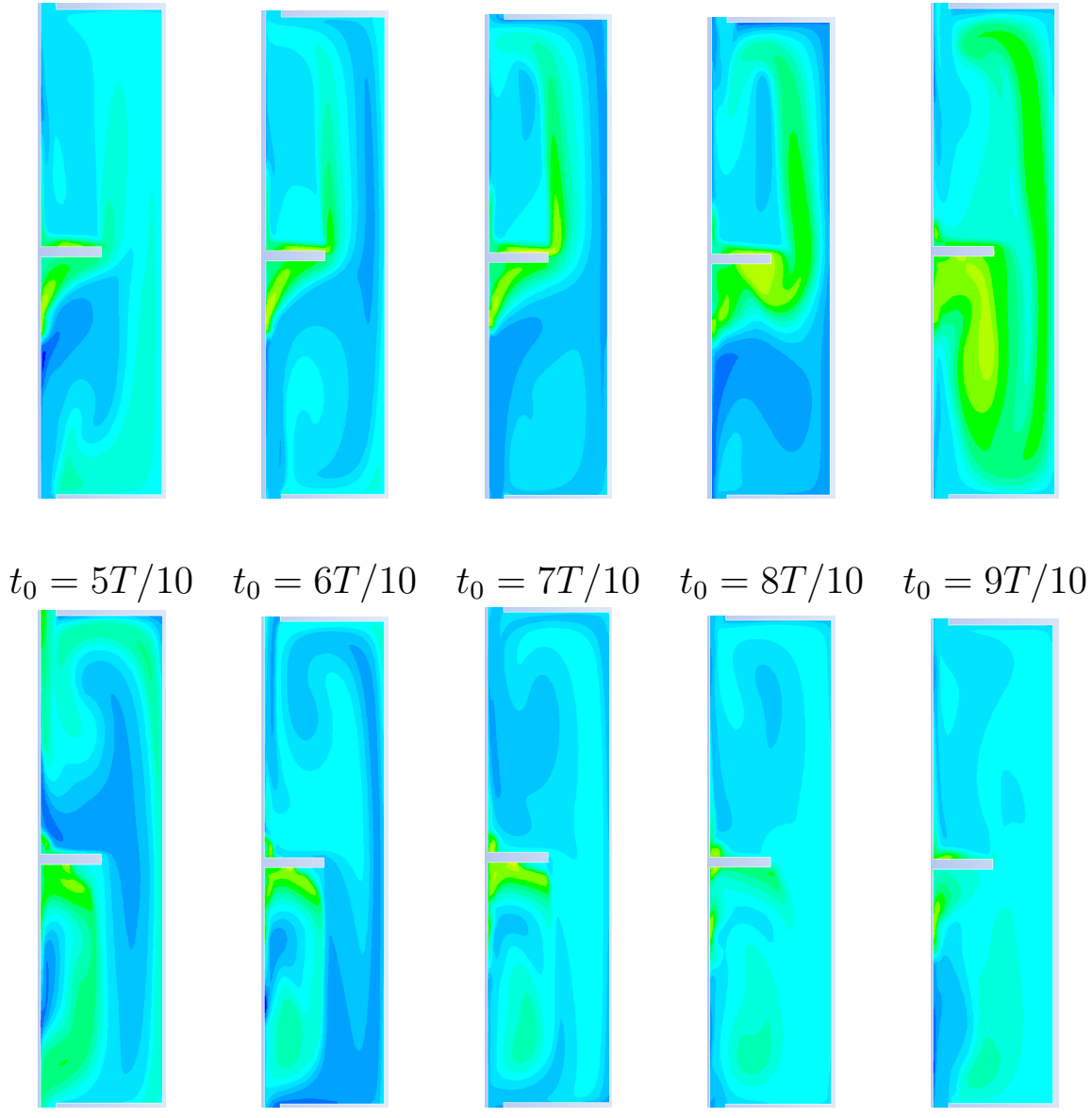

$t_{0}=9 T / 10$

Figure 7: Time evolution of the mean diameter $d_{32}$ during one pulsation period, $T$, in a compartment of the pulsed colomn for $A=60 \mathrm{~mm}, f=1 \mathrm{~Hz}$ (color code: $d_{32}$ value increases from $1.2 \mathrm{~mm}$ to $\approx 0$, i.e. no drops, from yellow to dark blue). The column axis is in the right. The compartement is bounded by two disks with a doughnut in the central part. 


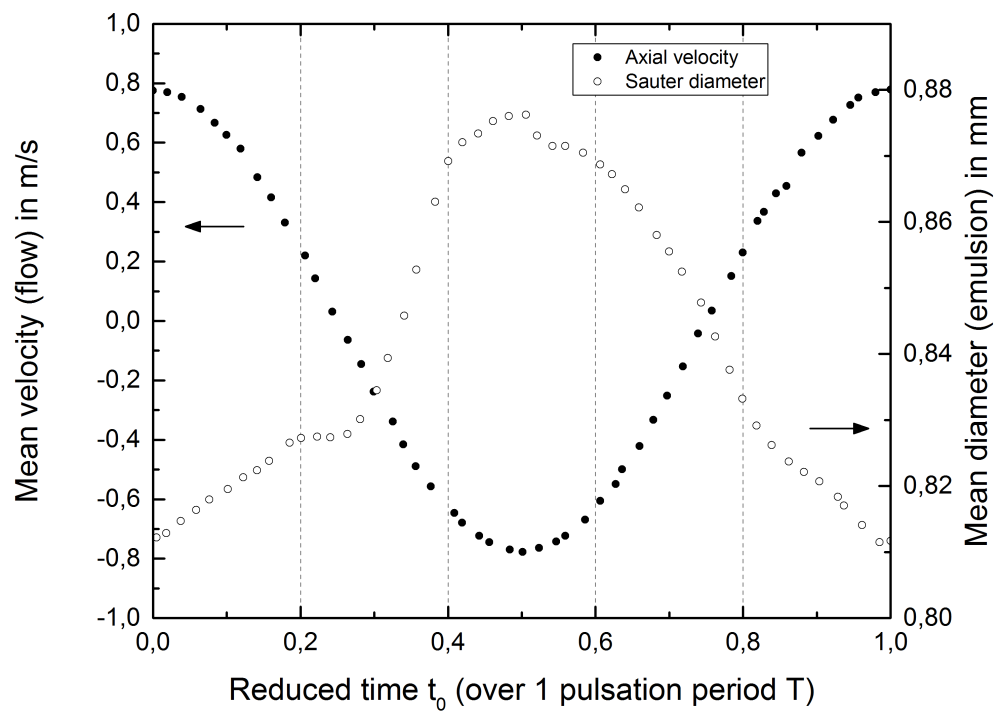

Figure 8: Simulated evolutions of the mean axial velocity and of the mean Sauter diameter, $d_{32}$, in the centerline of one compartement, over one pulsation period ( $A=60 \mathrm{~mm}, f=1$ $\mathrm{s}^{-1}$, phase system $\mathbf{I}$ ). 


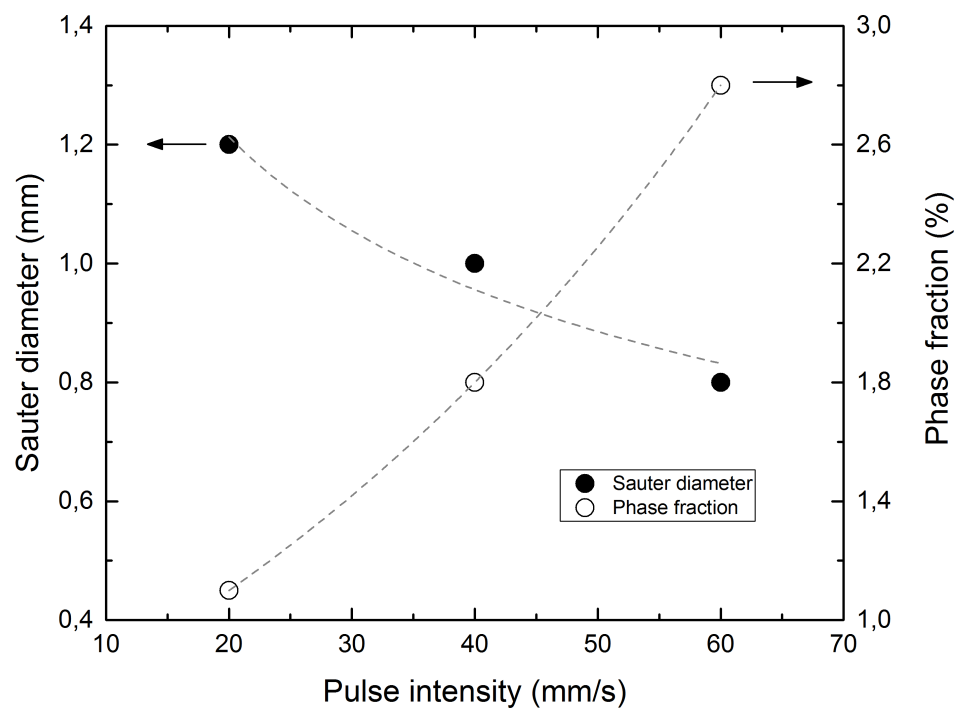

Figure 9: Model prediction for the evolution of $d_{32}$ and $\phi$ with increasing pulse intensity $(f=1 \mathrm{~Hz})$. Initial conditions $d_{32}=1.22 \mathrm{~mm}-\phi=2 \%$ (Symbols: simulation results; Lines: overall trends) 
literature. However, according to our experimental results, $A$ and $f$ seem to have a separated effect on $d_{32}$.

Seperated effects of $A$ and $f$ on the pulsed column hydrodynamics have already been reported in the literature in one-phase flow [42], where each individual parameter was shown to influence the residence time and trajectories of small particles, either droplets or solids. We also evidenced this effect in our previous parametric investigation of droplets residence time distribution [43]. However, distinct effect of the pulsation period and amplitude on the emulsification process in a pulsed column has, to our knowledge, never been reported so far.

Additionnal simulations were performed in order to address this point. The following configuration was considered: $A \times f=60 \mathrm{~mm} / \mathrm{s}, 0.5 \leq f \leq 2$ $\mathrm{Hz}$, initial diameter $d_{32}=1.22 \mathrm{~mm}$. As highlighted by Figure 10, the mean diameter calculated for the frequencies of 0.5 and $1 \mathrm{~s}^{-1}$ are very close, as it was observed experimentally for a similar breakup dominated case (run 5 and 10). However, imposing a significantly higher frequency $(f=2 \mathrm{~Hz})$ results in a drastic reduction of the droplet diameter. This could not be verified experimentally since this high $f$ value could not be achieved with the pulsing system we used. It is likely however that at $f=2 \mathrm{~Hz}$ the oscillating flow has not enough time to relaminarize between two direction changes, hence maintaining a high turbulence levels, and therefrom promoting breakage.

The hold-up on the contrary seems to be less sensitive to the variation of the pulsation frequency. This result is in agreement with the experimental observations. 


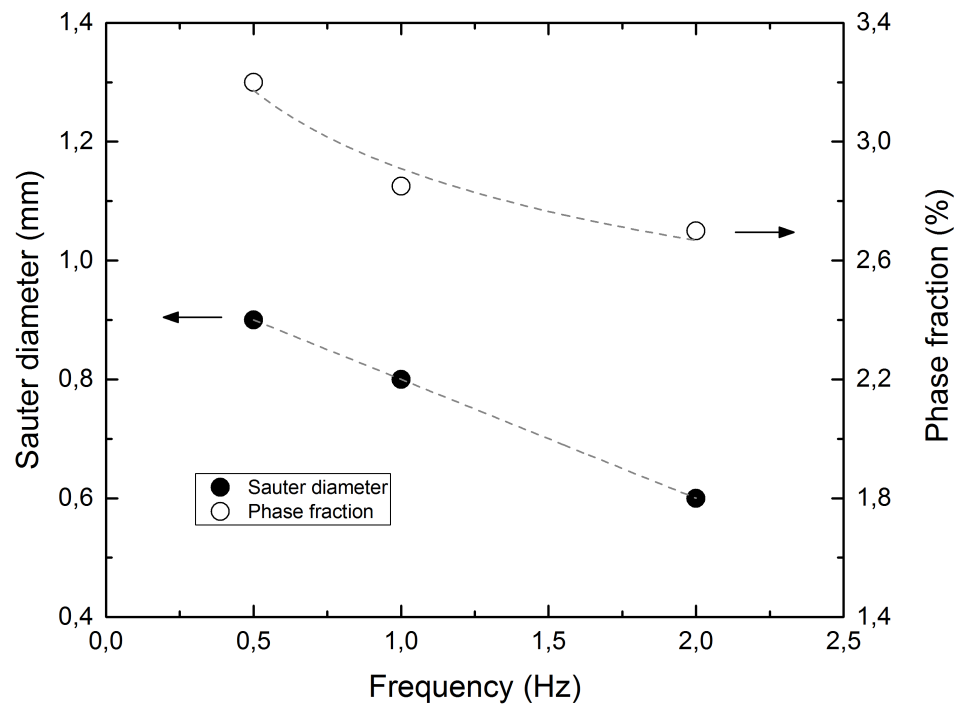

Figure 10: Separate effect of $A$ and $f$ on $d_{32}$ at $A \times f=60 \mathrm{~mm} \cdot \mathrm{s}^{-1}$ according to the model predictions. Initial conditions $d_{32}=1.22 \mathrm{~mm}$ (Symbols: simulation results; Lines: overall trends) 


\begin{tabular}{|c|c|c|c|}
\hline \multirow{2}{*}{ Phase system $(\mathrm{W} / \mathrm{O})$} & viscosity ratio & Surface tension & $d_{32}$ \\
\cline { 2 - 4 } & $\mu_{O} / \mu_{W}(-)$ & $(\mathrm{mN} / \mathrm{m})$ & $(\mathrm{mm})$ \\
\hline Phase system I & 1.2 & 43 & 0.84 \\
\hline$\downarrow$ & 13.2 & 43 & 0.32 \\
\hline Phase system II & 13.2 & 29 & 0.25 \\
\hline
\end{tabular}

Table 5: Influence of fluid properties at $60 \mathrm{~mm} / \mathrm{s}$ - operating conditions run $3 \mapsto$ run 4

Model relevance for high oil viscosity

Although the Coulaloglou and Tavlarides model is not valid for viscous breakage, simulations have been conducted in order to evaluate the model behaviour if the continuous phase exhibits higher viscosity. Indeed, during solvent extraction operations, the fluid properties may evolved notably along the axial direction of the column, as the elements to be recovered are extracted by the solvent, thus inducing an increase of the oil phase viscosity. The properties chosen for phase system (II) are typical of conditions locally encountered in the nuclear fuel cycle, where the solvent properties are sensitive to the content in heavy ions such as uranium. Hence, although extrapolating the turbulent breakage kernel to conditions where viscous breakage is expected to prevail has no scientific justification, it is nonetheless interesting from a chemical engineer point of view to investigate if this model would be appropriate for the simulation of an extraction column, where the fluid properties are varying. Hence the predictions of the breakage model, with the parmameters determined experimentally on liquid-liquid system (I) for which turbulent breakage dominates, have been examined for the experiments carried with system (II). The simulation results are reported in Table 5.

The numerical model predicts a decrease in the mean drops diameter with the viscosity of the continuous phase. This is consistent with the film 
drainage theory on which the coalescence kernel relies: the higher the fluid viscosity in the film, the higher the drainage time, and the lower the collision efficiency. However, as stated earlier, the results presented here are only qualitative as the model was intended for turbulent breakage, while the viscous system will rather undergo viscous shear breakage mechanisms.

As expected, the decrease of the interfacial tension results in a decrease in the mean diameter as the breakage is facilitated by the lower surface energy.

\section{Summary and Conclusion}

Following the development of a CFD-PBE coupled model of the liquidliquid flow in a pulsed column [19], on-line emulsion monitoring has been implemented in small diameter pulsed columns dedicated to R\&D studies. A parametric study was achieved in order to assess the emulsion properties sensitivity to specific operating conditions provided by the oscillating flow. Meaningful results have been obtained that allowed us to propose a breakage and coalescence model convenient for the simulation of the dispersed phase behaviour in a pulsed column.

Thanks to this model, the main features of the emulsification process in the pulsed column have been reproduced numerically, especially the effect of the pulsation intensity, and the individual effect of the pulse frequency on the achieved mean drop diameter has been confirmed. Moreover, thanks to the identified constants, the model is able to represent the breakage as well as the coalescence, which was not the case for the previously published parameters in the literature. Indeed, the predicted hold-up and $d_{32}$ evolutions were very satisfactory both qualitatively and quantitatively. At last, although the 
values of the kernels parameters were adjusted on a "low" viscosity waterin-oil emulsion, the model appeared to behave well with a higher viscous oil.

This numerical tool will allow reliable parametric numerical experiments to be performed and correlations of the emulsion properties to the column operating conditions to be derived. Such correlations are indeed needed to enlarge the validity of chemical engineering models that predict the separation performances, while reducing the need for time consuming flow resolution. In this aim, the study has to be enlarged to other chemical systems and extraction apparatus.

Anyway, the improved breakage and coalescence kernels we proposed remain empirical and there is still a long way before purely phenomelogical models, compatible with CFD simulations, be available. Besides the local flow / interface coupling and interfacial dynamics aspects, that are the aim of many works [44], there are still many specificities to be addressed regarding emulsification in liquid-liquid extraction processes. Among them, some are already the focus of research such as the effect of mass transfer and ionic stregnth on the coalescence efficiency $[45,46]$.

\section{Acknowledgement}

This work was supported by the Nuclear Energy Division of CEA (program DISN/AFCOE). 


\section{References}

[1] K. Gonda, T. Matsuda, Solvent extraction calculation model for PUREX process in pulsed sieve plate column, Journal of Nuclear Science and Technology 23 (10) (1986) 883-895.

[2] A. B. Jahya, G. W. Stevens, H. R. C. Pratt, Pulsed disc-and-doughnut column performance, Solvent Extraction and Ion Exchange 27 (1) (2009) 63-82.

[3] M. Jaradat, M. Attarakih, H.-J. Bart, Advanced prediction of pulsed (packed and sieve) extraction columns performances using population balance modelling, Chemical Engineering Research and Design 89 (12) (2010) 2752-2760.

[4] M. Montuir, C. Sorel, V. Pacary, X. Heres, H. Roussel, P. Baron, B. Dinh, PAREX simulation code: an efficient tool to model and simulate solvent extraction operations, in: P. SFGP (Ed.), Recents progrès en génie des procédés, vol. 101, 2011.

[5] M. Attarakih, A.-K. Mazen, H.-J. Bart, Modeling and dynamic analysis of a rotating disc contactor (RDC) extraction column using one primary one secondaray particle method (OPOSPM), Chemical Engineering Science 91 (2013) 180-196.

[6] A. Kumar, S. Harland, A unified correlation for the predication of dispersed-phase hold-up in liquid-liquid extraction columns, Industrial Engineering Chemistry Research 34 (1995) 3925-3940. 
[7] A. Kumar, S. Harland, Unified correlations for the predication of drop size in liquid-liquid extraction columns, Industrial Engineering Chemistry Research 35 (1996) 2682-2695.

[8] O. Syll, I. Mabille, M. Moscosa-Santillan, M. Traore, J. Amouroux, Study of mass transfer and determination of drop size distribution in a pulsed extraction column, Chemical Engineering Research and Design 89 (2011) 60-68.

[9] S. A. Schmidt, M. Simon, M. Attarakih, G. Lagar, H.-J. Bart, Droplet population balance modelling - hydrodynamics and mass transfer, Chemical Engineering Science 61 (2006) 246-256.

[10] N. B. Wyatt, T. J. O'Hern, B. Shelden, Drop-size distributions and spatial distributions in annular centrifugal contactor, AIChE Journal 59 (6) (2013) 2219-2226.

[11] M. Nakase, K. Takeshita, Numerical and experimental study on oilwater dispersion in a new countercurrent centrifugal extractor, Procedia Chemistry 7 (2012) 288-294.

[12] D. Garthe, Fluid dynamics and mass transfer of single particles and swarms of particles in extraction columns, Ph.D. thesis, Technische Universität München, 2006.

[13] E. Lobry, T. Lasuye, C. Gourdon, X. C., Liquid-liquid dispersion in continuous oscillatory baffled reactor - Application to suspension polmerization, Chemical Engineering Journal 259 (2015) 505-518. 
[14] A. Khalil, F. Puel, Y. Chevalier, J.-M. Galvan, A. Rivoire, J.-P. Klein, Study of droplet size distribution during an emulsification process using in situ video probe coupled with an automatic image analysis, Chemical Engineering Journal 165 (2010) 957.

[15] S. Maaß, J. Rojahn, R. Hänsch, M. Kraume, Automated drop detection using image analysis for online particle size monitoring in multiphase systems, Computers \& Chemical Engineering 45 (2012) 27-37.

[16] M. M. M. Ribeiro, C. Gonçalves, P. F. Regueiras, M. M. L. Guimarães, J. J. C. Cruz Pinto, Measurements of toluene-water dispersions hold-up using a non-invasive ultrasonic technique, Chemical Engineering Journal 118 (2006) 47-54.

[17] A.-M. O'Rourke, P.-F. MacLoughlin, A comparison of measurement techniques used in analysis of evolving liquid-liquid dispersions, Chemical Engineering and Processing 44 (2005) 885-894.

[18] S. Maaß, S. Wollny, A. Voigt, M. Kraume, Experimental comparison of measurement techniques for drop size distributions in liquid/liquid dispersions, Experiments in Fluids 50 (2011) 259-269.

[19] A. Amokrane, S. Charton, N. Sheibat-Othman, J. Becker, J.-P. Klein, F. Puel, Development of a CFD-PBE coupled model for the simulation of the drops behavior in a pulsed column, The Canadian Journal of Chemical Engineering 92 (2014) 220-233.

[20] N. Verrier, S. Cotmellec, M. Brunel, D. Lebrun, Digital in-line holog- 
raphy in thick optical systems: application to visualization in pipes, Applied optics 47 (22) (2008) 4147-4157.

[21] A. W. Pacek, I. P. T. Moore, A. W. Nienow, R. V. Calabrese, Video technique for measuring dynamics of liquid-liquid dispersion during phase inversion, AIChE Journal 40 (12) (1994) 1940-1949.

[22] B. Hu, P. Angeli, O. K. Matar, C. J. Lawrence, G. F. Hewitt, Evaluation of drop size distribution from chord length measurements, AIChE Journal 52 (3) (2006) 931-939.

[23] J. A. Boxall, C. A. Koh, E. D. Sloan, A. K. Sum, D. T. Wu, Measurement and calibration of droplet size distributions in Water-in-Oil emulsions by PVM and FBRM, Industrial \& Engineering Chemistry Research 49 (3) (2010) 1412-1418.

[24] A. Amokrane, S. Maaß, H. Roussel, F. Lamadie, S. Charton, In-situ droplets monitoring in a lab-scale pulsed column, in: International Solvent Extraction Conference ISEC, Würzburg, 2014.

[25] J. Illingworth, J. Kittler, A survey of the Hough transform, Computer vision, graphics and image processing 44 (1988) 87-116.

[26] M. Mickler, B. Boecker, H.-J. Bart, Drop swarm analysis in dispersions with incident-light and transmitted-light illumination, Flow Measurement and Instrumentation 30 (2013) 81-89.

[27] M. Mickler, S. Didas, M. Jaradat, M. Attarakih, H.-J. Bart, Droplet swarm analysis by image processing for simulation of extraction columns 
with population balances (in German), Chemie Ingenieur Technik 83 (3) (2011) 227-236.

[28] F. B. Alban, S. Sajjadi, M. Yianneskis, Dynamic tracking of fast liquidliquid dispersion processes with a real-time in-situ optical technique, Chemical Engineering Research and Design 82 (A8) (2004) 1054-1060.

[29] L. M. R. Brás, E. F. Gomes, M. M. M. Ribeiro, M. M. L. Guimarães, Drop distribution determination in a liquid-liquid dispersion by image processing, International Journal of Chemical Engineering (Article ID 746439) (2009) 6.

[30] R. Gopalan, D. Jacobs, Comparing and combining lighting insensitive approaches for face recognition, Computer Vision and Image Understanding 114 (1) (2010) 135-145.

[31] J. P. Lewis, Fast Normalized Cross-Correlation, Vision Interface (1995) $120-123$.

[32] C. A. Coulaloglou, L. L. Tavlarides, Description of interaction processes in agitated liquid-liquid dispersions, Chemical Engineering Science 32 (1977) 1289-1297.

[33] M. A. Hsia, L. L. Tavlarides, A simulation model for homogeneous dispersion in stirred tanks, Chemical Engineering Journal 20 (1980) 225236.

[34] P. M. Bapat, L. L. Tavlarides, Mass transfer in a liquid-liquid CFSTR, AIChE Journal 31 (1985) 659-666. 
[35] J. per Becker, F. Puel, Y. Chevalier, N. Sheibat-Othman, Monitoring silicone oil droplets during emulsification in stirred vessel: Effect of dispersed phase concentration and viscosity, The Canadian Journal of Chemical Engineering 92 (2) (2014) 296-306.

[36] K. J. Valentas, O. Bilous, N. R. Amundson, Analysis of breakage in dispersed phase systems, Industrial and Engineering Chemistry Fundamentals 5 (1966) 1103-1111.

[37] A. Amokrane, S. Charton, F. Lamadie, F. Paisant, J.-F. Puel, Singlephase flow in a pulsed column: Particle Image Velocimetry validation of a CFD based model, Chemical Engineering Science 114 (2014) 40-50.

[38] D. L. Marchisio, J. T. Pikturna, R. O. Fox, R. D. Vigil, A. A. Barresi, Quadrature method of moments for population balance equations, AIChE Journal 49 (2003) 1266-1276.

[39] D. L. Marchisio, R. D. Vigil, R. O. Fox, Implementation of the quadrature method of moment in CFD codes for agregation-breakage kernels, Chemical Engineering Science 58 (2003) 3337-3351.

[40] J. Solsvik, J. per Becker, N. Sheibat-Othman, I. Mohallick, R. Farzad, H. A. Jakobsen, Viscous drop breakage in liquid-liquid stirred dispersions: Population balance modeling, Journal of Dispersion Science and Technology 36 (2015) 577-594.

[41] C. Martinez-Bazan, J. L. Montanes, J. C. Lasheras, On the breakup of an air bubble injected into fully developed turbulent flow. Part 1: breakup frequency, Journal of Fluid Mechanics 401 (1999) 157-182. 
[42] M.-S. Aoun-Nabli, P. Guiraud, C. Gourdon, Numerical experimentations: a tool to calculate the axial dispersion coefficient in discs and doughnuts pulsed solvent extraction columns, Chemical Engineering Science 52 (1997) 2353-22368.

[43] A. Amokrane, S. Charton, F. Lamadie, J. Becker, J.-P. Klein, F. Puel, Study of the dispersed phase behavior in a pulsed column for oxalate precipitation in emulsion, 9th International conference on CFD in the mineral and process industries, Melbourne, Australia, 2012.

[44] J. Podgórska, W. Bałdyga, Scale-up effects on the drop size distribution in liquid-liquid disepersions in agitated vessels, Chemical Engineering Science 56 (2001) 741-746.

[45] J. Kamp, M. Kraume, Influence of drop size and superimposed mass transfer on coalescence in liquid/liquid dispersions - Test cell design for single drop invesigations, Chemical Engineering Research and Design 92 (2014) 635-643.

[46] J. Villwock, F. Gebauer, J. Kamp, H.-J. Bart, M. Kraume, Systematic Analysis of Single Droplet Coalescence, Chemical Engineering and Technology 37 (2014) 271-279. 


\section{Appendix 1: Note on the PBE-CFD model}

Computational domain and boundary conditions

The computational domain (e.g. number of compartments, mesh convergence, grid refinement at the wall) has been defined and validated in the hydrodynamic study performed in a $50 \mathrm{~mm}$ diameter column [37]. The same features have been used for the $25 \mathrm{~mm}$ column considered in this study. The boundary conditions for the continuous phase are similar too.

Regarding the dispersed phase, periodic conditions have been considered. To run the QMOM method, the emulsion properties, including the first 6 moments of the distributions, have to be initialized in the whole domain. Given the initial value of the hold-up $\phi$ and of the mean diameter $d_{0}$, and assuming mono-dispersed droplets at the initial step, the $0^{\text {th }}$ order momentum $m_{0}$, expressing the number of droplets $\left(\right.$ in $^{-3}$ ) was deduced from the

following relation: $d_{0}=\left(\frac{6 \phi}{\pi m_{0}}\right)^{1 / 3} \cdot \phi$ and $m_{0}$ are therefore initialized given the conditions prevailing in the column before the change in the operating conditions (either measured experimentally or converged values from a previous simulation, depending on the case studied). The other momentums are deduced from $m_{0}$ as explained in the manuscript.

Note that it is also possible to initialize the solution thanks to a source term of dispersed phase at a given location of the computationnal domain, as we did in our previous study [19].

\section{Turbulence model}

The $k-\epsilon$ model ability to tackle the pulsed flow turbulent properties, including the turbulent dissipation $\epsilon$ required for the source terms of the 


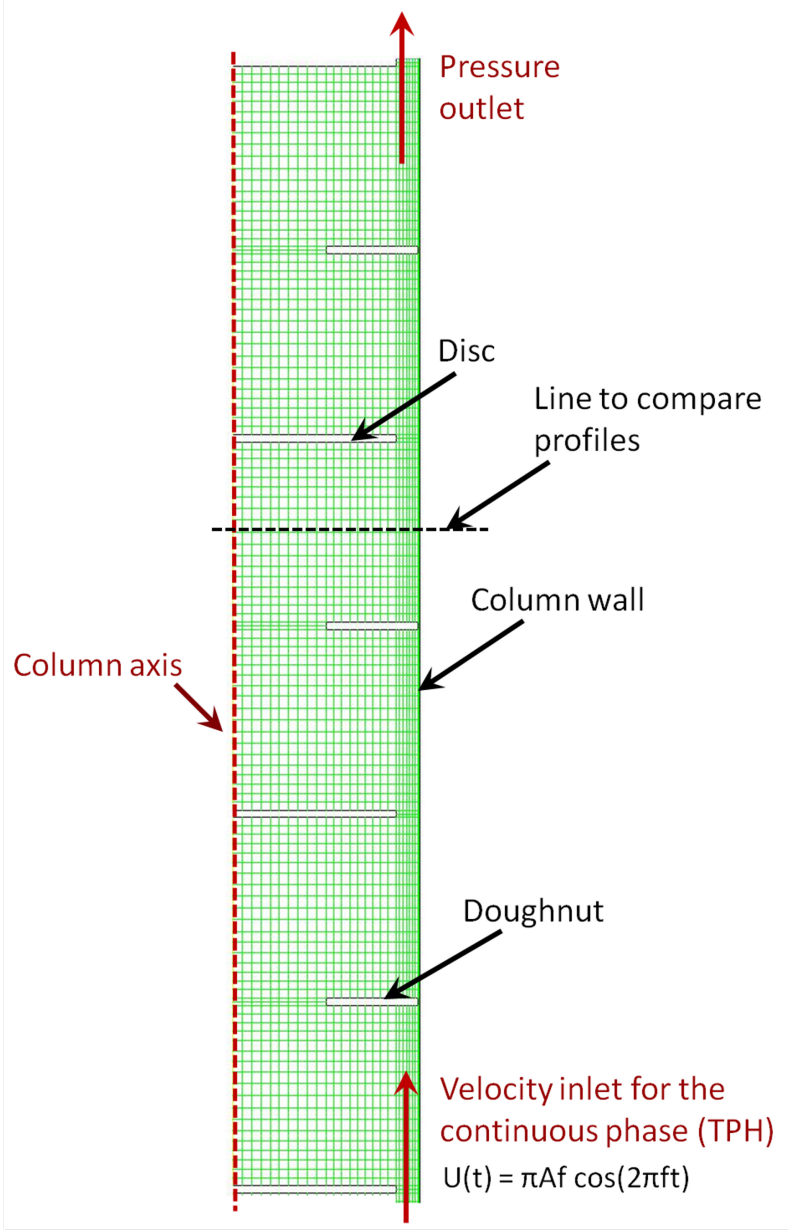

Figure 11: Computational domain and flow boundary conditions (from [37]) 
population balance, was highlighted in our previous paper [37] thanks to PIV measurements synchronized on the flow period.

Yet, while the $k-\epsilon$ model appeared to behave correctly in most cases, some deviations between the model and the experimental values were observed at the 2 time-steps corresponding to abrupt changes in the flow direction, as depicted on Fig. 12. This discrepancy was also observed if a non isotrope turbulence model is considered. Indeed, no RANS model is able to accurately predict $\epsilon$ at these particular moments of the flow period, i.e. the fast flow transients, which is not surprising. However, since LES is much less suitable for routine CFD-PBE simulations, we believe that the $k-\epsilon$ model is accurate enough to be coupled with the considered breakage and coalescence kernels, that are not able to track the interface dynamic neither.

\section{Convergence behaviour}

The convergence of the CFD-PBE simulations was checked regarding the dispersed phase average diameter, volume fraction and moments. In order to tackle convergence issues, that could be observed when the coalescence source term is activated, the first order upwind scheme was chosen.

Moreover, while a time step of $1 \mathrm{~ms}$ is required in order to have a Courant Friederichs Lewy number (CFL) lower than 1, and suffice to perform accurate hydrodynamic simulations, the time step has to be reduced for the CFD-PBE simulations to fix numerical issues. A time step of $\delta t=10^{-4} \mathrm{~s}$ was used for all the CFD-PBE simulations. An exemple of simulation results is given on Fig. 13. The evolution of the Sauter diameter from the initial to the converged value, following an increase of the pulsation intensity, is depicted, thus allowing the convergence trend to be appreciated. The experimental 

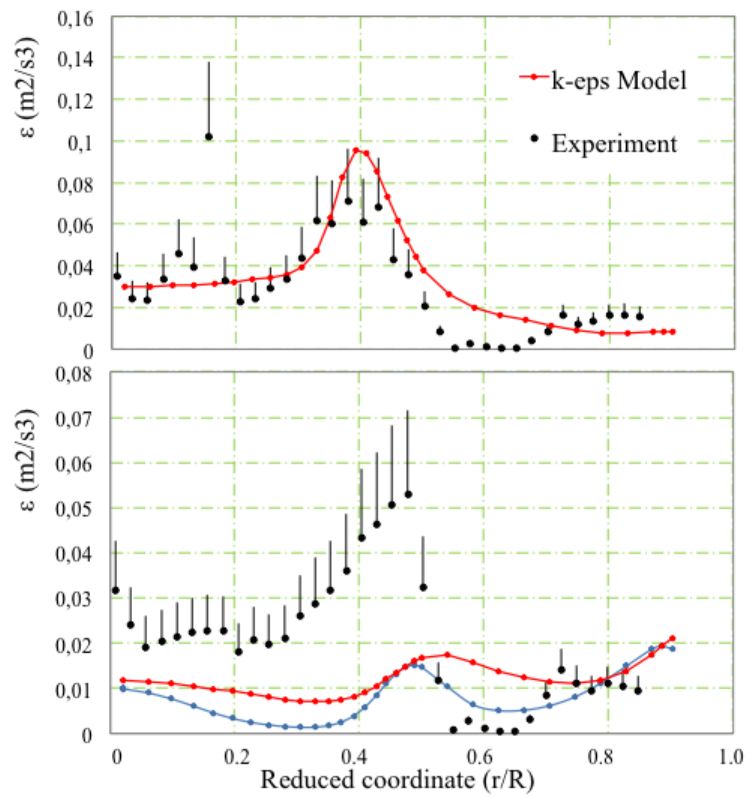

Figure 12: Typical $\epsilon$ profiles in the pulsed column. Comparison of experimental and numerical results at a moment where the flow evolution is monotonic (top) and at the moment of abrupt change of direction (bottom). Note that the four "zero" values in the experimental curves at approx $r / R=0.6$ are not representative, since the PIV particles are hidden behing the column packing at this particular locations 


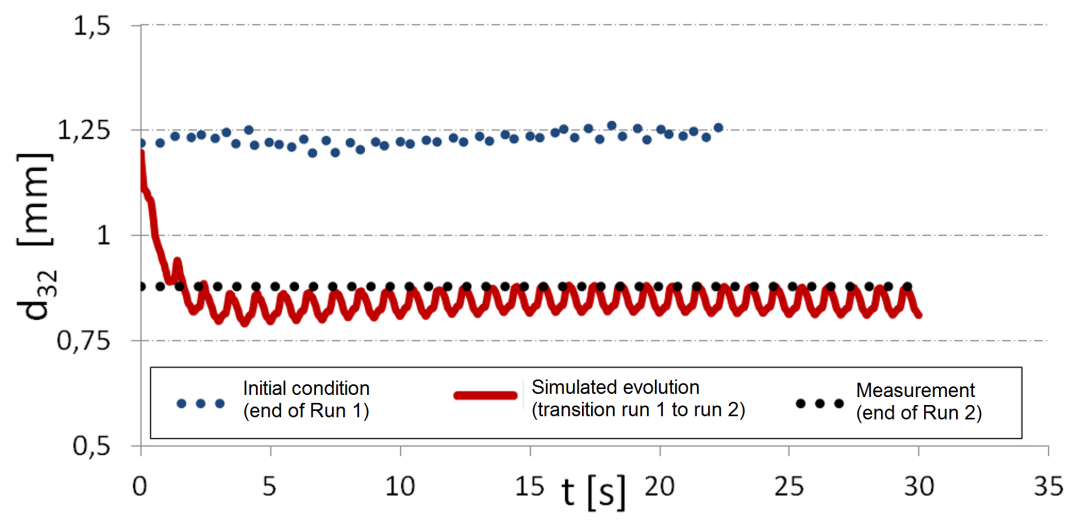

Figure 13: Transient behaviour predicted by the model for the transition between $A=40 \mathrm{~mm} f=1 \mathrm{~Hz}$ (run 1) and $A=60 \mathrm{~mm} f=1 \mathrm{~Hz}$ (run 2), and comparison with experimental data at equilibrium.

value is also reported on the graph for the sake of comparison.

\section{Appendix 2: Comment on the parameters identification}

The simplified procedure for the parameters identification was the following.

1. In our previous paper [19] a first set of parameters able to describe both the coalescence and the breakage in the water in oil system has been determined. The identification procedure was based on the dynamic evolution of the DSD in a stirred tank reactor, monitored by an in-situ video probe. The Finite Volumes method was used to solve the PBE, and numerical values of the parameters $C_{1}$ to $C_{4}$ have been deduced by an inverse method. Besides the selection of appropriate kernels and parameters identification, this first stage enabled us to estimate the emulsion sensitivy to the 4 parameters $C_{1}, C_{2}, C_{3}$ and $C_{4}$ of the model. 


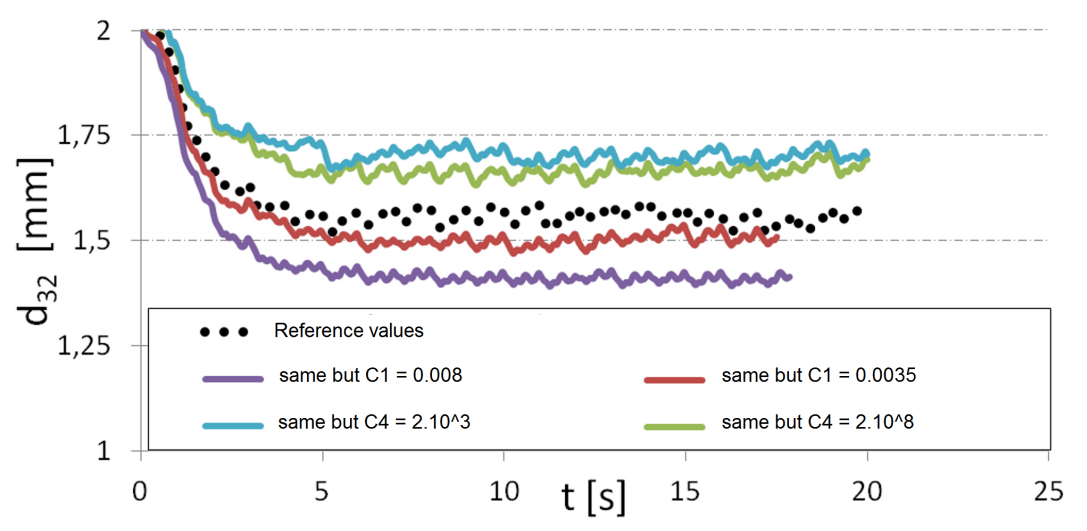

Figure 14: Sensitivity of the simulation results to the numerical value of the constants $C_{1}$ and $C_{4}$, respectively affecting breakage and coalescence. Reference values are: $C_{1}=0.00487 ; C_{2}=0.0552 ; C_{3}=2.1710^{-4} ; C_{4}=2.2810^{13}[32]$. Considered hydrodynamic conditions: $f=1 \mathrm{~Hz}$ and $A=4 \mathrm{~mm}$.

2. From these conclusions regarding the PBE model alone, and after the time and grid scales convergence was checked, we examined the coupled PBE-CFD model sensitivity by changing drastically the value of each $C_{i}$ independently. Exemple of result are presented in Fig. 14 where each curve corresponds to the modification of one parameter compare to the reference Coulaloglou and Tavlarides model. It can be observed that increasing the breakage rate (respectively the coalescence) induces a reduction (respectively an increase) of the mean drop diameter, which highlights the model ability to depict both phenomena.

3. Given the hudge variations considered, the preliminary simulations pointed out that the numerical model is robust towards these paramaters, and that the emulsification process we consider is weakly sensitive to the $C_{i}$ constants. The latter observations reinforced us in our "coarse" methodology for the parameters adjustment, instead of a more rigorous identification process, that would have been tedious 
considering the coupled CFD-PBE problem.

4. Then, the coarse iterative process described in the manuscript has been implemented. 